\title{
Evaluating the snappability of bar-joint frameworks
}

\author{
G. Nawratil
}

\begin{abstract}
It is well-known that there exist bar-joint frameworks (without continuous flexions) whose physical models can snap between different realizations due to nondestructive elastic deformations of material. We present a method to measure these snapping capability - shortly called snappability - based on the total elastic strain energy of the framework by computing the deformation of all bars using Hooke's law. The presented theoretical results give further connections between shakiness and snapping beside the well-known technique of averaging and deaveraging.
\end{abstract}

Key words: Snapping framework, multistability, model flexor, elastic deformation

\section{Introduction}

We consider a framework in the Euclidean $n$-dimensional space $\mathbb{E}^{n}$ which consists of a knot set $\mathscr{K}=\left\{K_{1}, K_{2}, \ldots, K_{s}\right\}$ and an abstract graph $G$ on $\mathscr{K}$ fixing the combinatorial structure. We denote the edge connecting $K_{i}$ to $K_{j}$ by $e_{i j}$ with $i<j$ and collect all indices of knots edge-connected to $K_{i}$ in the knot neighborhood $N_{i}$. Moreover we denote the number of edges in the graph by $b$ and assign 1 a length $L_{i j} \in \mathbb{R}_{>0}$ to each edge $e_{i j}$. In general this assignment does not determine the shape of the framework uniquely thus a framework has different incongruent realizations. For example, a triangular framework has in general two realizations in $\mathbb{E}^{2}$, which are not congruent with respect to the group of direct isometries. If we consider the isometry group then this number halves.

By materializing all edges by straight bars and linking them by $S_{n}$-joint $\mathrm{s}^{2}$ in the

Institute of Discrete Mathematics and Geometry \& Center for Geometry and Computational Design, TU Wien, Austria, e-mail: nawratilegeometrie.tuwien.ac.at

${ }^{1}$ This assignment corresponds to the definition of the intrinsic metric of the framework.

${ }^{2} S_{n}$ denotes the spherical joint, which enables the group of spherical motions $\operatorname{SO}(n)$ of $\mathbb{E}^{n}$. Note that a $S_{2}$-joint equals a rotational joint (R-joint). 
knots, we end up with a so-called bar-joint framework. We assume that (1) all bars are uniform made of a homogeneous isotropic material deforming at constant volume (i.e. Poisson's ratio $v=1 / 2$ ) and that (2) all $S_{n}$-joints are without clearance.

In the following we consider the configuration of knots $\mathbf{k}:=\left(\mathbf{k}_{1}, \ldots, \mathbf{k}_{s}\right)$, where $\mathbf{k}_{i}$ denotes the $n$-dimensional coordinate vector of the knot $K_{i}$, which together with the graph $G$ implies the framework's realization $G(\mathbf{k})$. In the rigidity community (e.g. [2]) each edge $e_{i j}$ is assigned with a stress (coefficient) $\omega_{i j} \in \mathbb{R}$. If in every knot $i \in\{1, \ldots, s\}$ the so-called equilibrium condition

$$
\sum_{i<j \in N_{i}} \omega_{i j}\left(\mathbf{k}_{i}-\mathbf{k}_{j}\right)+\sum_{i>j \in N_{i}} \omega_{j i}\left(\mathbf{k}_{i}-\mathbf{k}_{j}\right)=\mathbf{o}
$$

is fulfilled, where $\mathbf{o}$ denotes the $n$-dimensional zero-vector, then the $b$-dimensional vector $\omega=\left(\ldots, \omega_{i j}, \ldots\right)^{T}$ is refereed as self-stress (or equilibrium stress). According to Gluck [4] and Roth [15] the existence of a non-zero self-stres ${ }^{3}$ corresponds to the infinitesimal flexibility (shakiness) of the framework's realization $G(\mathbf{k})$.

Shakiness (of order on ${ }^{4}$ ) can also be seen as the limiting case where two realizations of a framework coincide [18, 20, 22]. In contrast a realization $G\left(\mathbf{k}^{\prime}\right)$ is called a snapping realization if it is close enough to another incongruent realization such that the physical model can snap (flip/jump) into this realization due to non-destructive elastic deformations of material. The open problem in this context is the meaning of closeness, which is tackled in this article. In more detail, we present a method to measure the snapping capability (shortly called snappability) of a realization $G\left(\mathbf{k}^{\prime}\right)$, based on the total elastic strain energy of the framework.

Before we plunge in medias res we provide a short review on snapping (also called multistable; cf. [5]) structures. During the last years the interest in these structures has increased due to practical applications (e.g. [14, 7, 17]).

It is pointed out in [20] that there is a direct connection between shakiness and snapping through the technique of averaging and deaveraging, respectively (cf. [16, page 1604] and [8]). The latter allows to construct snapping frameworks in any dimension. Moreover for snapping bipartite frameworks in $\mathbb{E}^{n}$ an explicit result in terms of confocal hyperquadrics is known (cf. [20, page 112] under consideration of [19]). Most results are known for the dimension $n=3$, which are summarized next.

There is a series of papers of Walter Wunderlich on snapping spatial structures (octaeder, Bennett mechanisms, antiprisms, icosaeder, dodecaeder), which are reviewed in [18]. In this context also the paper [5] should be cited, where buckling polyhedral surfaces and Siamese dipyramids are introduced. Snapping structures are also related to so-called model flexor ${ }^{5}$ (cf. [11]) as in some cases the model flexibility can be reasoned by the snapping through different realizations. Examples for this phenomenon are the so-called four-horn [23] or the already mentioned

\footnotetext{
${ }^{3} \omega$ differs from the $b$-dimensional zero vector.

${ }^{4}$ Each additional coinciding realization raises the order of the infinitesimal flexibility by one [22].

${ }^{5}$ Mathematically these structures do not posses a continuous flexibility but due to free bendings without visible distortions of materials their physical models flex.
} 
Siamese dipyramids. The latter are studied in more detail in [6], especially how relative variations on the edge lengths produce significant relative variations in the spatial shape. The authors of [6] also suggested estimates to quantify these intrinsic and extrinsic variations.

\section{Physical model of deformation}

First we consider a single bar $e_{i j}$ and apply equal but opposite directed forces $F_{i j}$ to it's ends pointing outwards/inwards the bar, which imply a tensile/compression stress leading to an expansion/decrease of the bar. According to Hooke's law, which can be applied due to the elastic deformation during the process of snapping, the tensile/compression stress $\delta_{i j}$ in a uniform bar equals the product of the modulus of elasticity $\left[E_{i j}>0\right.$ and the Cauchy/engineering strain ${ }^{7} \varepsilon_{i j}$; i.e.

$$
\delta_{i j}=E_{i j} \varepsilon_{i j} \quad \text { with } \quad \varepsilon_{i j}=\frac{l_{i j}-L_{i j}}{L_{i j}} \quad \text { for } \quad i<j
$$

where $l_{i j} \in \mathbb{R}_{>0}$ is the deformed length (stressed length) of the bar $e_{i j}$, while $L_{i j}$ is the original length (unstressed length) of the bar $e_{i j}$. Note that $\delta_{i j}>0$ corresponds to a stretching and $\delta_{i j}<0$ to a compression. Taking Eq. (2) into account the wellknown relation $F_{i j}=\delta_{i j} A_{i j}$, where $A_{i j}$ is the cross-sectional area of the bar $e_{i j}$, can be rewritten as

$$
F_{i j}=E_{i j} A_{i j} \frac{l_{i j}-L_{i j}}{L_{i j}} \text { for } i<j .
$$

This force acts on the end points $K_{i}$ and $K_{j}$ of the involved bar $e_{i j}$ by the force vectors

$$
\mathbf{f}_{i j}=F_{i j} \frac{\mathbf{k}_{i}-\mathbf{k}_{j}}{\left\|\mathbf{k}_{i}-\mathbf{k}_{j}\right\|} \quad \text { and } \quad \mathbf{f}_{j i}=-\mathbf{f}_{i j}
$$

where $\|$.$\| denotes the standard norm. Moreover, the elastic strain energy { }^{8}$ stored in the bar $e_{i j}$ can be computed by

$$
U_{i j}=\frac{1}{2} F_{i j}\left(l_{i j}-L_{i j}\right) \stackrel{\sqrt[3]{3}}{\longrightarrow} U_{i j}=\frac{1}{2} \frac{E_{i j} A_{i j}}{L_{i j}}\left(l_{i j}-L_{i j}\right)^{2}
$$

according to [12, page 512], where $E_{i j} A_{i j} / L_{i j}$ is the axial stiffness of the bar $e_{i j}$. As a consequence the total deformation energy $U$ of the framework reads as

$$
U:=\sum_{i<j} U_{i j}=\frac{1}{2} \sum_{i<j} \frac{E_{i j} A_{i j}}{L_{i j}}\left(l_{i j}-L_{i j}\right)^{2} .
$$

\footnotetext{
${ }^{6}$ In this paper we assume $E_{i j}>0$ as for conventional structural material $E_{i j}$ is positive.

${ }^{7}$ Equals in this case the engineering normal strain $\varepsilon_{i j}$ of a material line segment $e_{i j}$.

${ }^{8}$ Note that elastic strain energy is a form of potential energy.
} 
Remark 1. In the study of rigidity and stability of frameworks similar functions appear; e.g. the function

$$
\Phi=\frac{1}{2} \sum_{i<j} \frac{F_{i j}}{L_{i j}}\left(l_{i j}^{2}-L_{i j}^{2}\right)
$$

was used by Kötter [9]. Connelly and Whiteley [3] based their results on a potential energy function $\sum_{i j} H_{i j}\left\|\mathbf{k}_{i}-\mathbf{k}_{j}\right\|^{2}$ where $H_{i j}$ is any real-valued function of one variable, which has a local minimum at $\left\|\mathbf{k}_{i}-\mathbf{k}_{j}\right\|^{2}$.

Expressing $l_{i j}$ in dependence of the knots $K_{i}$ and $K_{j}$ the system of partial derivatives

$$
\frac{\partial U}{\partial k_{i, 1}}=0, \quad \frac{\partial U}{\partial k_{i, 2}}=0, \quad \cdots, \quad \frac{\partial U}{\partial k_{i, n}}=0,
$$

where $\left(k_{i, 1}, \ldots, k_{i, n}\right)$ is the coordinate vector of $\mathbf{k}_{i}$, equals the condition $\sum_{j \in N_{i}} \mathbf{f}_{i j}=\mathbf{o}$ for $i=1, \ldots, s$. With respect to our physical model the meaning of the real values $\omega_{i j}$ in Eq. 11 is $F_{i j} / l_{i j}$, which is the so-called force density with respect to the stressed length [21]. Moreover it should be pointed out that each critical point of the total elastic strain-energy $U(\mathbf{k})$ corresponds to a self-stressed framework realization $G(\mathbf{k})$, which is called deformed for $U(\mathbf{k})>0$ and undeformed for $U(\mathbf{k})=0$.

Remark 2. The system of equations $\sum_{j \in N_{i}} \mathbf{f}_{i j}=\mathbf{o}$ was also obtained by Linkwitz and Schek [10, page 149ff.], who studied the form finding problem for cable networks. Within their proposed force density method [21] values are assigned to the mentioned force densities rendering these equations linear in the knot coordinates.

\subsection{Metric interpretation of $U$}

This physical model implies in the $b$-dimensional space $\mathbb{R}^{b}$ of bar lengths the following scalar product $\langle., .\rangle_{P}: \mathbb{R}^{b} \times \mathbb{R}^{b} \rightarrow \mathbb{R}$ with $(\mathbf{x}, \mathbf{y}) \mapsto\langle\mathbf{x}, \mathbf{y}\rangle_{P}:=\mathbf{x}^{T} P \mathbf{y}$ and

$$
\mathbf{x}:=\left(\begin{array}{c}
\vdots \\
x_{i j} \\
\vdots
\end{array}\right), \quad \mathbf{y}:=\left(\begin{array}{c}
\vdots \\
y_{i j} \\
\vdots
\end{array}\right), \quad P:=\left(\begin{array}{ccc}
\ddots & & \\
& \frac{E_{i j} A_{i j}}{2 L_{i j}} & \\
& & \ddots
\end{array}\right),
$$

as the involved $b \times b$ diagonal matrix $P$ is positive definite. Consequently this scalar product induces a norm $\|\cdot\|_{P}: \mathbb{R}^{b} \rightarrow \mathbb{R}_{\geq 0}$ with $\mathbf{x} \mapsto\|\mathbf{x}\|_{P}:=\sqrt{\langle\mathbf{x}, \mathbf{x}\rangle_{P}}$ and a metric (distance function) $d_{P}(.,):. \mathbb{R}^{b} \times \mathbb{R}^{b} \rightarrow \mathbb{R}_{\geq 0}$ with $(\mathbf{x}, \mathbf{y}) \mapsto d_{P}(\mathbf{x}, \mathbf{y}):=\|\mathbf{x}-\mathbf{y}\|_{P}$. Thus $U$ of Eq. (6) can be seen as squared distance between the deformed framework $\mathbf{l}=$ $\left(\ldots, l_{i j}, \ldots\right)^{T}$ and the initial one $\mathbf{L}=\left(\ldots, L_{i j}, \ldots\right)^{T}$; i.e. $U=d_{P}(\mathbf{l}, \mathbf{L})^{2}$. It should be pointed out that this distance only depends on the intrinsic metric of the framework and is therefore independent of the actual realization.

In order to reduce the distance measure $d_{P}$ to its geometric core we set $E_{i j}=1$ and $A_{i j}=A$ for all bars in the remainder of the article. 


\section{Snappability of realizations}

If we have a realization $G(\mathbf{k})$ with non-zero self-stress and the corresponding total elastic energy $U(\mathbf{k})$ is not at a local minimum, then small perturbations would deform the framework according to the minimum total potential energy principle. Therefore we are interested in the set $\mathscr{S}$ of stable realizations, i.e. realizations which are at a local minimum of the total elastic strain-energy. Note that the set $\mathscr{S}$ is not empty as it contains at least the undeformed framework realizations.

\subsection{Computation of the set $\mathscr{S}$}

For the computation of the set $\mathscr{S}$ for a given framework with a given intrinsic metric the following approach is used. We introduce new variables $q_{i j}$ fulfilling the side condition $\Lambda_{i j}=0$ with $\Lambda_{i j}:=q_{i j}^{2}-\left\|\mathbf{k}_{i}-\mathbf{k}_{j}\right\|^{2}$ and make the Lagrange ansatz

$$
F(\mathbf{k}, \mathbf{q}, \lambda):=\frac{A}{2} \sum_{i<j} \frac{1}{L_{i j}}\left(q_{i j}-L_{i j}\right)^{2}+\sum_{i<j} \lambda_{i j} \Lambda_{i j}
$$

with the $b$-dimensional vectors $\mathbf{q}:=\left(\ldots, q_{i j}, \ldots\right)^{T}$ and $\lambda:=\left(\ldots, \lambda_{i j}, \ldots\right)^{T}$, where the latter is composed of the Lagrange multipliers $\lambda_{i j}$. By taking the partial derivatives of $F$ with respect to the $(s n+2 b)$ variables $(\mathbf{k}, \mathbf{q}, \lambda)$ we obtain a system of equations, which is of algebraic nature. Therefore we can use homotopy continuation method (e.g. Bertini; cf. [1]), as other approaches (e.g. Gröbner base, resultant based elimination) are not promising due to the number of unknowns and degree of equations. First of all we can restrict to the obtained real critical points of $F$ with all $q_{i j}>0$ as only these correspond to realizations. This resulting set $\mathscr{R}$ of realizations is split into a set $\mathscr{M}$ and its absolute complement $\mathscr{M}^{c}=\mathscr{R} \backslash \mathscr{M}$, where the elements of $\mathscr{M}$ correspond to local minima of $U(\mathbf{k})$. They can be identified by the so-called second derivative test; i.e. all eigenvalues of the Hessian matrix of the function $U(\mathbf{k})$ are positive. Finally the desired set $\mathscr{S}$ can be obtained as the quotient $\mathscr{M} / \mathrm{SE}(n)$, where $\mathrm{SE}(n)$ denotes the group of direct isometries of $\mathbb{E}^{n}$. In the same way we define the set $\mathscr{S}^{c}:=\mathscr{M}^{c} / \mathrm{SE}(n)$ of unstable realizations, which is needed later on.

\subsection{Measuring the snappability}

The evaluation of the snappability has to be based on the intrinsic metric of the framework, as minor changes of this metric can heavily effect its spatial shape (cf. examples of the four-horn [23] and Siamese dipyramids [6]). Our intrinsic metric approach towards the determination of the snappability of an undeformed realization is based on the following theorem: 
Theorem 1. If a framework snaps out of a stable realization $G\left(\mathbf{k}^{\prime}\right)$ by applying the minimum energy needed to it, then the corresponding deformation of the realization has to pass a shaky realization $G\left(\mathbf{k}^{\prime \prime}\right)$ at the maximum state of deformation.

Proof. We think of $U$ as a graph function over the space $\mathbb{R}^{s n}$ of knots $\mathbf{k}$; i.e. the ordered pair $(\mathbf{k}, U(\mathbf{k}))$. In order to get out of the valley of the local minimum $\left(\mathbf{k}^{\prime}, U\left(\mathbf{k}^{\prime}\right)\right)$, which corresponds to the given stable realization $G\left(\mathbf{k}^{\prime}\right) \in \mathscr{S}$, with a minimum of energy needed, one has to pass a saddle point $\left(\mathbf{k}^{\prime \prime}, U\left(\mathbf{k}^{\prime \prime}\right)\right)$ of the graph, which corresponds to a realization $G\left(\mathbf{k}^{\prime \prime}\right) \in \mathscr{S}^{c}$. As all realizations of $\mathscr{S}^{c}$ are selfstressed and deformed ( $\Rightarrow$ no zero self-stress) they are infinitesimally flexible.

Based on the pseudometric $d_{S}: \mathbb{R}^{b} \times \mathbb{R}^{b} \rightarrow \mathbb{R}_{\geq 0}$ with $\left(\mathbf{I}^{\prime}, \mathbf{I}^{\prime \prime}\right) \mapsto \mid d_{p}\left(\mathbf{I}^{\prime \prime}, \mathbf{L}\right)^{2}-$ $d_{p}\left(\mathbf{I}^{\prime}, \mathbf{L}\right)^{2} \mid$ the snappability index $s\left(\mathbf{k}^{\prime}\right)$ of $G\left(\mathbf{k}^{\prime}\right) \in \mathscr{S}$ can be quantified as follows

$$
s\left(\mathbf{k}^{\prime}\right)=\frac{\left|U\left(\mathbf{k}^{\prime \prime}\right)-U\left(\mathbf{k}^{\prime}\right)\right|}{A L}=\frac{\left|U\left(\mathbf{I}^{\prime \prime}\right)-U\left(\mathbf{I}^{\prime}\right)\right|}{A L}=\frac{d_{S}\left(\mathbf{l}^{\prime}, \mathbf{I}^{\prime \prime}\right)}{A L}
$$

where $\mathbf{l}^{\prime}=\left(\ldots, l_{i j}^{\prime}, \ldots\right)^{T} \in \mathbb{R}^{b}$ and $\mathbf{l}^{\prime \prime}=\left(\ldots, l_{i j}^{\prime \prime}, \ldots\right)^{T} \in \mathbb{R}^{b}$ are the bar lengths of the $G\left(\mathbf{k}^{\prime}\right) \in \mathscr{S}$ and $G\left(\mathbf{k}^{\prime \prime}\right) \in \mathscr{S}^{c}$, respectively, using the notation of the above proof and $L=\sum_{i<j} L_{i j}$ denotes the framework's total length. Note that due to the division by the framework's volume $A L$, which is constant according to the assumed Poisson's ratio $v=1 / 2$, the snappability index $s\left(\mathbf{k}^{\prime}\right)$ can be interpreted as the change of the elastic strain energy density $U /(A L)$. Therefore $s\left(\mathbf{k}^{\prime}\right)$ is invariant with respect to scaling (taking into account $E_{i j}=1$; cf. Sec. 2.1p and enables the comparison of frameworks, which differ in the number of knots, the combinatorial structure and intrinsic metric. Note that the minimum of the obtained snappability indices over all undeformed realizations can be seen as the snappability index of the framework.

Before we give the algorithm for computing the snappability index $s(\mathbf{k})$ of an undeformed realization $G(\mathbf{k})$, it should be noted that the concrete curve $\left(\mathbf{k}_{t}, U\left(\mathbf{k}_{t}\right)\right)$ on the graph connecting $(\mathbf{k}, 0)$ and the saddle point $\left(\mathbf{k}^{\prime \prime}, U\left(\mathbf{k}^{\prime \prime}\right)\right)$ does not play a role as long as the deformation energy $U_{i j}$ of each bar $e_{i j}$ is monotonic increasing with respect to the curve parameter $t$. This is due to the fact that along each curve of this possible set $\mathscr{C}$ of curves the same amount of mechanical work (namely the minimum work needed) is performed on the framework to reach the saddle point.

Algorithm. Given is an undeformed realization $G(\mathbf{k})$. Let us assume that the unstable realization $G\left(\mathbf{k}^{\prime \prime}\right) \in \mathscr{S}^{c}$ yields the minimal function value $U\left(\mathbf{k}^{\prime \prime}\right)$. We consider the simplest possible path in $\mathbb{R}^{b}$, namely the straight line segment from $\mathbf{L}$ to $\mathbf{l}^{\prime \prime}$ and parametrize it with respect to the time $t \in[0,1]$ yielding $\mathbf{I}_{t}:=\mathbf{L}+t\left(\mathbf{I}^{\prime \prime}-\mathbf{L}\right)$. This path corresponds to different 1-parametric deformations of realizations in $\mathbb{E}^{n}$. If among these a deformation $G\left(\mathbf{k}_{t}\right)$ with the property

$$
\left.G\left(\mathbf{k}_{t}\right)\right|_{t=0}=G(\mathbf{k}),\left.\quad G\left(\mathbf{k}_{t}\right)\right|_{t=1}=G\left(\mathbf{k}^{\prime \prime}\right)
$$

exists $\left(\Rightarrow\left(\mathbf{k}_{t}, U\left(\mathbf{k}_{t}\right)\right) \in \mathscr{C}\right)$, then the undeformed realization $G(\mathbf{k})$ can be left over the unstable realization $G\left(\mathbf{k}^{\prime \prime}\right)$ and we get a value for $s(\mathbf{k})$. Computationally the property (12) can be checked e.g. by a parameter homotopy approach (e.g. Bertini; 
cf. [1, Sec. 6]). If such a deformation does not exist then we redefine $\mathscr{S}^{c}$ as $\mathscr{S}^{c} \backslash$ $\left\{G\left(\mathbf{k}^{\prime \prime}\right)\right\}$ and run again the procedure explained in this paragraph until we end up with a value for $s(\mathbf{k})$. In the case of $\mathscr{S}^{c}=\varnothing$ we set $s(\mathbf{k})=\infty$.

Remark 3. Note that our computational approach using Bertini does not recognize if the tracked path between the real starting point and real endpoint of the homotopy is entirely real. More generally it is an open problem to check if there exists at least one real curve $\in \mathscr{C}$, which corresponds to such a continuous real deformation.

After applying the mechanical minimum work needed to deform $G\left(\mathbf{k}^{\prime}\right)$ into $G\left(\mathbf{k}^{\prime \prime}\right)$ as described in Theorem 1 the framework will relax according to the minimum total potential energy principle. Therefore this self-acting deformation will end up in a stable realization or it get stuck on the way to such a local minimum by reasons of reality. Note that a realization at the boarder of reality has to be infinitesimal flexible, as a real solution of an algebraic set of equations can only change over into a complex one through a double root. This results in the following theorem:

Theorem 2. A snap of a framework described in Theorem 1 ends up in a realization $G\left(\mathbf{k}^{\prime \prime \prime}\right)$ which is either undeformed or a deformed one with a shakiness.

Remark 4. Note that $s\left(\mathbf{k}^{\prime}\right)$ is independent of the final snapping realization $G\left(\mathbf{k}^{\prime \prime \prime}\right) . \diamond$

\subsection{Pinned frameworks}

From the mechanical point of view it makes sense to fix a system to the ground by pinning a subset $\mathscr{P}$ of the knot set $\mathscr{K}$. The results of this paper also hold for this scenario of so-called pinned graphs (or grounded graphs) due to their fundamental properties summarized in [13, Sec. 2.1]. One only has to keep in mind that bars between pinned knots cannot be deformed and that the equilibrium condition (1) only has to hold for knots $\in \mathscr{K} \backslash \mathscr{P}$ as pinned knots can counterbalance any force. This becomes clear by studying a trivial example (comparison of a pinned and unpinned triangular framework) given in the Appendix (cf. Sec. 5.1), where the computation of the snappability index is also demonstrated for a more sophisticated framework (pinned 3-legged planar parallel manipulator; cf. Sec. 5.2).

\section{Conclusion and open problems}

The total elastic strain energy of the framework (based on a physical model for the deformation of bars using Hooke's law) serves as base for the presented snappability index and the theoretical results of Theorem 1 and 2 , which give further connections between shakiness and snapping beside the technique of averaging and deaveraging.

Note that our approach neglects the possibility of collision of bars during the framework's deformation. A further open problem is mentioned in Remark 3 . 
Acknowledgements The research is supported by Grant No. P 30855-N32 of the Austrian Science Fund FWF. Thanks to Hellmuth Stachel for constructive feedback on the final draft.

\section{References}

1. Bates, D.J., Hauenstein J.D., Sommese, A.J., Wampler C.W.: Numerically Solving Polynomial Systems with Bertini. SIAM Philadelphia (2013)

2. Connelly, R.: Rigidity. Handbook of Convex Geometry (P.M. Gruber, J.M. Wills eds.), pages 223-271, Elsevier (1993)

3. Connelly, R., Whiteley, W.: The Stability of Tensegrity Frameworks. International Journal of Space Structures 7(2) 153-163 (1992)

4. Gluck, H.: Almost all simply connected closed surfaces are rigid. Geometric Topology (L.C. Glaser, T.B. Rushing eds.), pages 225-239, Springer (1975)

5. Goldberg, M.: Unstable Polyhedral Structures. Mathematics Magazine 51(3) 165-170 (1978)

6. Gorkavyy, V., Fesenko, I.: On the model flexibility of Siamese dipyramids. Journal of Geometry 110:7 (2019)

7. Haghpanah, B., Salari-Sharif, L., Pourrajab, P., Hopkins, J., Valdevit, L.: Multistable ShapeReconfigurable Architected Materials. Advanced Materials 28(36) 7915-7920 (2016)

8. Izmestiev, I.: Statics and kinematics of frameworks in Euclidean and non-Euclidean geometry. Eighteen Essays in Non-Euclidean Geometry (V. Alberge, A. Papadopoulos eds.), pages 191233, EMS Publishing House (2019)

9. Kötter, E.: Über die Möglichkeit, $n$ Punkte in der Ebene oder im Raume durch weniger als $2 n-3$ oder $3 n-6$ Stäbe von ganz unveränderlicher Länge unverschieblich miteinander zu verbinden. Festschrift Heinrich Müller-Breslau (H. Boost et al eds.), pages 61-80, Alfred Kröner Verlag Leipzig (1912)

10. Linkwitz, K., Schek, H.-J.: Einige Bemerkungen zur Berechnung von vorgespannten Seilnetzkonstruktionen. Ingenieur-Archiev 40(3) 145-158 (1971)

11. Milka, A.D.: Linear bendings of right convex polyhedra. Matematicheskaya fizika, anliz, geometriya 1(1) 116-130 (1994)

12. Mittemeijer, E. J.: Fundamentals of Materials Science. Springer (2011)

13. Nixon, A., Schulze, B., Sljoka, A., Whiteley, W.: Symmetry Adapted Assur Decomposition. Symmetry 6(3) 516-550 (2014)

14. Rafsanjani, A., Akbarzadeh, A., Pasini, D.: Snapping Mechanical Metamaterials under Tension. Advanced Materials 27(39) 5931-5935 (2015)

15. Roth, B.: Rigid and Flexible Frameworks. The American Mathematical Monthly 88(1) 6-21 (1981)

16. Schulze, B., Whiteley, W.: Rigidity and scene analysis. Handbook of Discrete and Computational Geometry (J.E. Goodman et al eds.), pages 1593-1632, 3rd edition, CRC Press (2017)

17. Shang, X., Liu, L., Rafsanjani, A., Pasini, D.: Durable bistable auxetics made of rigid solids. Journal of Materials Research 33(3) 300-308 (2018)

18. Stachel, H.: W. Wunderlichs Beiträge zur Wackeligkeit. Technical Report No. 22, Institute of Geometry, TU Wien (1995)

19. Stachel, H.: Configuration theorems on bipartite frameworks. Rendiconti del Circolo Matematico di Palermo (Series 2) 70(II) 335-351 (2002)

20. Stachel, H.: What lies between rigidity and flexibility of structures. Serbian Architectural Journal 3(2) 102-115 (2011)

21. Tibert, A.G., Pellegrino, S.: Review of Form-Finding Methods for Tensegrity Structures. International Journal of Space Structures 26(3) 241-255 (2011)

22. Wohlhart, K.: Degrees of shakiness. Mechanism and Machine Theory 34(7) 1103-1126 (1999)

23. Wunderlich, W., Schwabe, C.: Eine Familie von geschlossenen gleichflächigen Polyedern, die fast beweglich sind. Elemente der Mathematik 41(4) 88-93 (1986) 


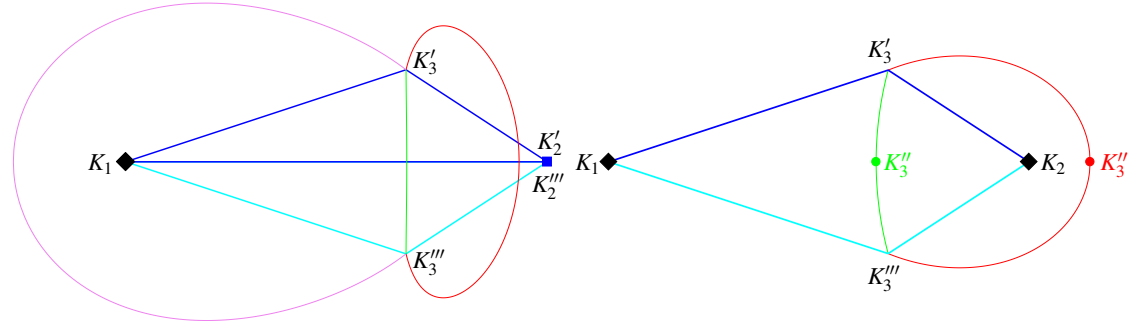

Fig. 1 Trajectories of the vertex $K_{3}$ during the snapping deformations (three in the unpinned case; two in the pinned case) from the blue into the cyan realization, which correspond to straight line segments in the space $\mathbb{R}^{b}$ of bar lengths. (right) Pinned case, where the pinned knots are indicated by the black diamonds. (left) Also in the unpinned case we can pin the first knot $K_{1}$ without loss of generality. During the snapping deformation the horizontal bar with endpoint $K_{2}$ (indicated by a blue square) changes its length, which is pointed out in Fig. 2

\section{Appendix of examples}

\subsection{Pinned and unpinned triangular framework}

We study the triangle with vertices $K_{1}, K_{2}, K_{3}$ and edge lengths $L_{12}=10, L_{13}=7$ and $L_{23}=4$. In the first approach we consider an unpinned framework where we attach the fixed frame in the following way to the framework: The origin coincide with $K_{1}$ and the positive $x$-axis points into direction of $K_{2}$; i.e.

$$
\mathbf{k}_{1}=(0,0), \quad \mathbf{k}_{2}=\left(k_{2,1}, 0\right), \quad \mathbf{k}_{3}=\left(k_{3,1}, k_{3,2}\right) .
$$

In the second approach we pin $K_{1}$ and $K_{2}$ yielding

$$
\mathbf{k}_{1}=(0,0), \quad \mathbf{k}_{2}=\left(L_{12}, 0\right), \quad \mathbf{k}_{3}=\left(k_{3,1}, k_{3,2}\right) .
$$

Unpinned case: According to the computation of Sec. 3.1, the set $\mathscr{S}$ consists of the following two undeformed realizations:

$$
\left(k_{2,1}, k_{3,1}, k_{3,2}\right)=\left(10, \frac{133}{20}, \frac{7}{20} \sqrt{39}\right), \quad\left(k_{2,1}, k_{3,1}, k_{3,2}\right)=\left(10, \frac{133}{20},-\frac{7}{20} \sqrt{39}\right),
$$

and the set $\mathscr{S}^{c}$ of the following three unstable realizations:

$$
\begin{array}{rlll}
\left(k_{2,1}, k_{3,1}, k_{3,2}\right) & =\left(\frac{20}{3}, \frac{28}{3}, 0\right) & \Longrightarrow & \left(l_{12}, l_{13}, l_{23}\right)=\left(\frac{20}{3}, \frac{28}{3}, \frac{8}{3}\right), \\
\left(k_{2,1}, k_{3,1}, k_{3,2}\right)=\left(\frac{80}{21},-\frac{8}{3}, 0\right) & \Longrightarrow & \left(l_{12}, l_{13}, l_{23}\right)=\left(\frac{80}{21}, \frac{8}{3}, \frac{136}{21}\right), \\
\left(k_{2,1}, k_{3,1}, k_{3,2}\right)=\left(\frac{220}{21}, \frac{20}{3}, 0\right) & \Longrightarrow & \left(l_{12}, l_{13}, l_{23}\right)=\left(\frac{220}{21}, \frac{20}{3}, \frac{80}{21}\right) .
\end{array}
$$

The two undeformed realizations and the trajectories of $K_{3}$ under the snapping deformations between them passing through the unstable realizations are illustrated in Fig. 11(left) and Fig. 22 respectively. Note that the snappability index 


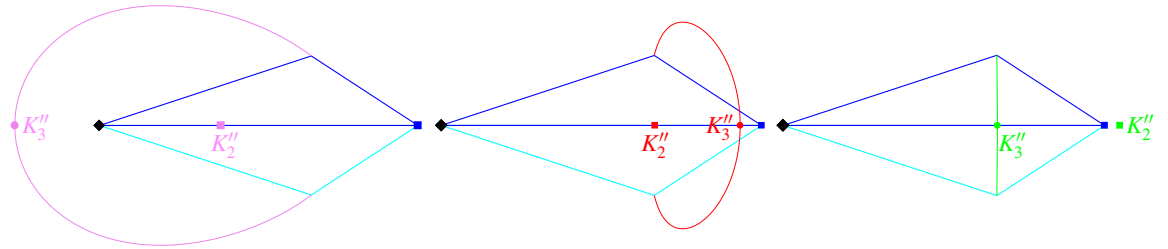

Fig. 2 Unstable realizations of the unpinned triangular framework: Each snapping deformation passes an unstable realization, which is displayed in violet (left), red (center) and green (right), respectively. The corresponding animations of these snapping deformations can be downloaded from www. dmg.tuwien.ac.at/nawratil/publications.html.

$s\left(10, \frac{133}{20}, \pm \frac{7}{20} \sqrt{39}\right)$ equals $1 / 882$, which corresponds to the elastic strain energy density of the green unstable realization. The corresponding values for the red and violet unstable realizations are 49/882 and $169 / 882$, respectively.

Pinned case: We obtain the same set $\mathscr{S}$ as in the unpinned case (cf. Eq. (15)), but the set $\mathscr{S}^{c}$ consists only of the following two realizations:

$$
\begin{aligned}
& \left(k_{3,1}, k_{3,2}\right)=\left(\frac{126}{11}, 0\right) \quad \Longrightarrow \quad\left(l_{13}, l_{23}\right)=\left(\frac{126}{11}, \frac{16}{11}\right), \\
& \left(k_{3,1}, k_{3,2}\right)=\left(\frac{70}{11}, 0\right) \quad \Longrightarrow \quad\left(l_{13}, l_{23}\right)=\left(\frac{70}{11}, \frac{40}{11}\right),
\end{aligned}
$$

which are illustrated in Fig. 11 right) together with the trajectories of $K_{3}$ under the two corresponding snapping deformations. For this trivial example the two saddle points of the graph of the elastic strain energy density function $U\left(k_{3,1}, k_{3,2}\right) /(A L)$ can even be visualized (see Fig. 3). The function values of the red and green saddle points are $49 / 462$ and $1 / 462$, respectively, where the latter one equals the snappability index $s\left(\frac{133}{20}, \pm \frac{7}{20} \sqrt{39}\right)$ of the pinned triangular framework.
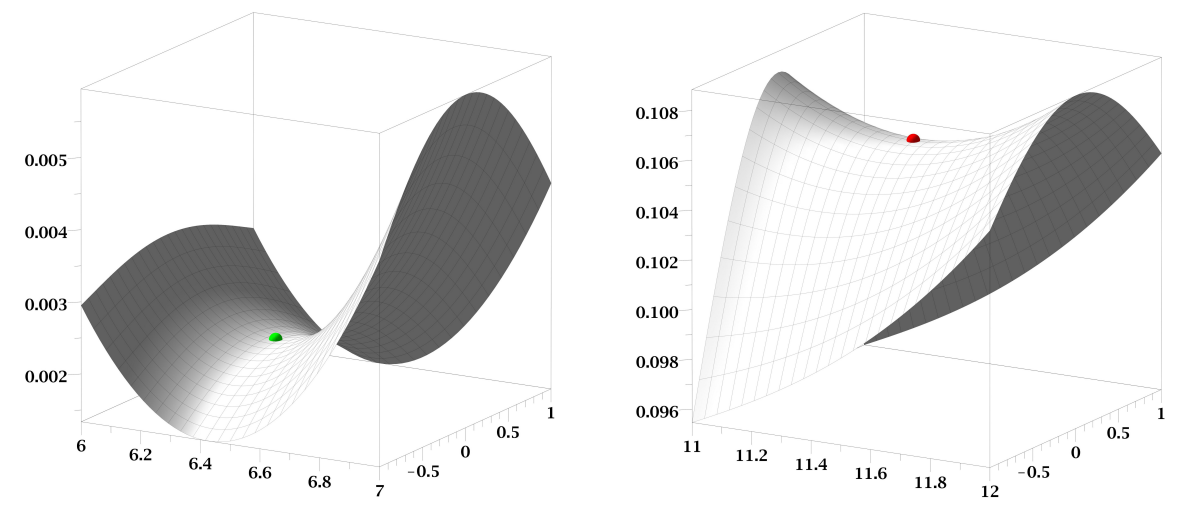

Fig. 3 Graph of the function $U\left(k_{3,1}, k_{3,2}\right) /(A L)$ of the pinned triangular framework: (left) $k_{3,1} \in$ $[6 ; 7]$ and $k_{3,2} \in[-1 ; 1]$ (right) $k_{3,1} \in[11 ; 12]$ and $k_{3,2} \in[-1 ; 1]$. The green and red saddle points correspond to the two unstable realization indicated in Fig. 1]right). 


\subsection{Pinned 3-legged planar parallel manipulator}

This more sophisticated framework (compared to the triangular one studied before) consists of six knots $K_{1}, \ldots, K_{6}$, where the first three knots are pinned to the ground possessing the following coordinates:

$$
\mathbf{k}_{1}=(0,0), \quad \mathbf{k}_{2}=(3,0), \quad \mathbf{k}_{3}=(2,1)
$$

Each of these knots $K_{i}$ is connected by a bar, the so-called $i$-th leg, with one of the remaining three knots $K_{i+3}$ (for $i=1,2,3$ ) with

$$
\mathbf{k}_{4}=\left(k_{4,1}, k_{4,2}\right), \quad \mathbf{k}_{5}=\left(k_{5,1}, k_{5,2}\right), \quad \mathbf{k}_{6}=\left(k_{6,1}, k_{6,2}\right),
$$

which form a joint-bar triangle (cf. Fig. 4). The intrinsic metric of the framework is given by:

$$
\left(L_{14}, L_{25}, L_{36}, L_{45}, L_{46}, L_{56}\right)=(4,5,3,3,1,2)
$$

and implies that the joint-bar triangle $K_{4}, K_{5}, K_{6}$ degenerates as $L_{46}+L_{56}=L_{45}$ holds. According to the computation of Sec. 3.1, the set $\mathscr{S}$ consists of the following three stable realizations:

$$
\begin{aligned}
& \left(\mathbf{k}_{4}, \mathbf{k}_{5}, \mathbf{k}_{6}\right)=((0.8876,3.9002),(-1.5278,2.1210),(0.0824,3.3071)), \\
& \left(\mathbf{k}_{4}, \mathbf{k}_{5}, \mathbf{k}_{6}\right)=((2.0771,3.4184),(4.8072,4.6619),(2.9871,3.8329)), \\
& \left(\mathbf{k}_{4}, \mathbf{k}_{5}, \mathbf{k}_{6}\right)=((2.9116,-2.4707),(0.3581,-3.8446),(1.8691,-2.2512)),
\end{aligned}
$$

where the first (blue) and second (cyan) are undeformed realizations and the third (magenta) is a deformed one (cf. Fig. 4(left)). The elastic strain energy density of the latter realization equals 0.00219 .

The set $\mathscr{S}^{c}$ contains 47 unstable realizations where the one with the smallest elastic strain energy density value of 0.00061 is given by:

$$
\left(\mathbf{k}_{4}, \mathbf{k}_{5}, \mathbf{k}_{6}\right)=((3.2050,2.5883),(1.4895,4.8801),(3.1261,3.6410)),
$$

which is illustrated in Fig. 4(right). The remaining 46 elements of $\mathscr{S}^{c}$ are given in Table 1 for reasons of completeness.

Remark 5. The critical points of the elastic strain energy function where computed by Bertini based on the splitting of the variables into the following two groups:

$\left(k_{4,1}, k_{4,2}, k_{5,1}, k_{5,2}, k_{6,1}, k_{6,2}, q_{14}, q_{25}, q_{36}, q_{45}, q_{46}, q_{56}\right), \quad\left(\lambda_{14}, \lambda_{25}, \lambda_{36}, \lambda_{45}, \lambda_{46}, \lambda_{56}\right)$,

which resulted in 59136 paths. In contrast the full homotopy yields 262144 paths. $\diamond$

The framework cannot snap out of the magenta realization by passing the green one, as the elastic strain energy density of the latter realization is lower. Therefore we consider in the space $\mathbb{R}^{6}$ of edge lengths the straight line segment $\mathbf{l}_{t}$ between the point given in Eq. 23, and the corresponding point of the green realization given by 


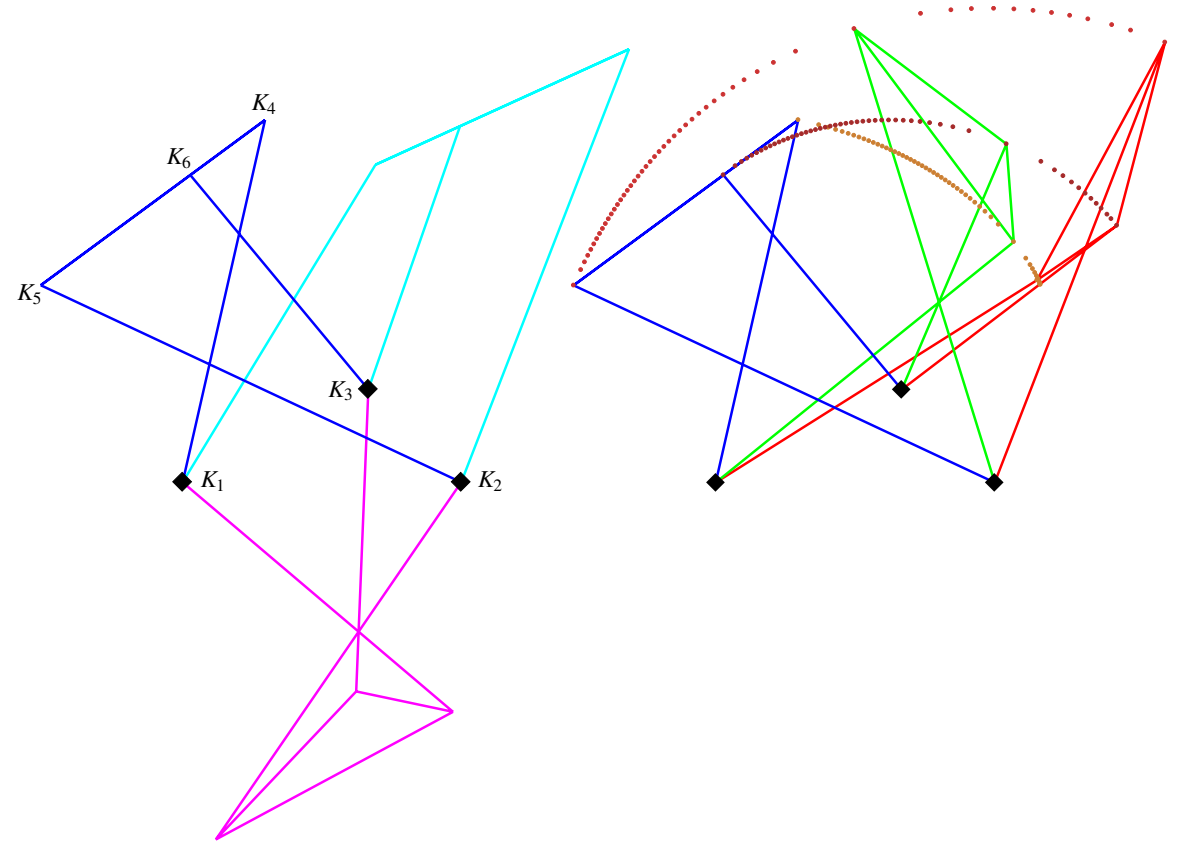

Fig. 4 (left) Illustration of the three stable realizations: the blue and the cyan realization are undeformed and the magenta one is deformed. The latter one is shaky as it is a deformed ( $\Rightarrow$ non-zero self-stress) stable realization. This can also be seen by the fact that the three legs belong to a pencil of lines. Note that in this example also the undeformed realizations are shaky due to the degenerated triangle $K_{4}, K_{5}, K_{6}$.

(right) Snapping deformation from the blue realization, passing through the green unstable realization and ending up at the red realization, which is on the boarder of reality. The line segment between the corresponding points of the blue and green realization (cf. Eqs. (23) and (26) in the space $\mathbb{R}^{6}$ of edge lengths is equally discretized ( 40 intervals). The same holds for the line segment between the corresponding points of the green realization and the complex one given in Eq. 27].

$$
\left(l_{14}, l_{25}, l_{36}, l_{45}, l_{46}, l_{56}\right)=(4.1196,5.1085,2.8710,2.8626,2.0527,1.0555) .
$$

One of the corresponding 1-parametric deformations has the property that it connects the blue realization with the green realization (cf. Eq. (12)). A further corresponding deformation also ends up in the green realization. This second deformation does not start at the cyan realization, but in the following complex solution for an undeformed realization of the framework:

$$
\begin{aligned}
\left(\mathbf{k}_{4}, \mathbf{k}_{5}, \mathbf{k}_{6}\right)=( & (3.8697-0.5179 i, 1.6591+1.2081 i),(6.9159+0.1526 i \\
& 3.1185-0.1916 i),(4.8851-0.2944 i, 2.1456+0.7415 i)) .
\end{aligned}
$$

Therefore the framework will relax from the green realization towards this complex solution. The realization, where this 1-parametric deformation hits the boarder of reality, is illustrated by the red shaky realization in Fig. 4(right). 


\begin{tabular}{|c|c|c|c|c|c|c|}
\hline$k_{4,1}$ & $k_{5,1}$ & $k_{6,1}$ & $k_{4,2}$ & $k_{5,2}$ & $k_{6,2}$ & $U /(A$ \\
\hline 4.030 & 6.239 & 4.489 & -0.5010 & -2.720 & -1.352 & 00058 \\
\hline-2.579 & .714 & -1.482 & -1.345 & 1.415 & -0.6494 & 0177 \\
\hline 0.9358 & 2.609 & 0.3781 & 3.889 & 4.985 & 3.524 & 0.0185 \\
\hline 1.179 & -0.7525 & 1.823 & 3.822 & 3.304 & 3.995 & 0.0185 \\
\hline 2.778 & 2.941 & 2.750 & -2.770 & -4.796 & -2.078 & 0191 \\
\hline-2.456 & -0.3583 & -1.199 & -1.645 & -3.345 & -1.294 & 0.0201 \\
\hline-2.156 & 0.1224 & -1.160 & 3.506 & 2.084 & 3.090 & 0.0204 \\
\hline 2.212 & 1.947 & 2.213 & 3.810 & 5.681 & 3.265 & 0.0290 \\
\hline 4.987 & 6.767 & 4.520 & 0.7908 & 2.181 & 0.5923 & 0.0315 \\
\hline-0.0774 & 0.9562 & -0.3930 & 3.889 & 2.578 & 4.320 & 0.0504 \\
\hline 1.822 & -1.357 & 0.5219 & 0.7588 & 1.022 & 0.1156 & 0.0512 \\
\hline 3.841 & 1.058 & 3.144 & 0.5499 & 1.821 & -0.1909 & 0.0585 \\
\hline 3.484 & 0.9825 & 3.365 & -0.9764 & 1.343 & -0.0521 & 0.0627 \\
\hline 3.717 & 1.094 & 3.193 & -0.7774 & 1.565 & -0.0846 & 0.0629 \\
\hline-2.140 & -3.594 & -1.331 & -0.2606 & -0.5004 & 0.2326 & 0.0633 \\
\hline-1.683 & -1.276 & -2.146 & -0.7357 & -3.000 & -0.7246 & 0.0685 \\
\hline-1.739 & -1.943 & -1.977 & -0.8342 & 1.398 & -1.090 & 0.0709 \\
\hline-1.903 & -1.030 & -0.4593 & 3.516 & 3.015 & 2.686 & 0.0740 \\
\hline-1.658 & -0.6953 & -0.0537 & 3.640 & 3.368 & 3.187 & 0.0741 \\
\hline-1.773 & -1.128 & -0.6979 & 3.586 & 2.821 & 2.312 & 0.0741 \\
\hline 3.046 & 0.6959 & 3.677 & -0.2001 & 0.9677 & -0.3291 & 0.0744 \\
\hline-1.042 & -0.5401 & -0.1441 & -3.448 & -2.650 & -1.908 & 0.0813 \\
\hline 1.789 & 2.145 & 2.256 & 3.171 & 4.195 & 4.650 & .0828 \\
\hline-2.847 & -1.875 & -1.217 & -1.478 & -0.9779 & -0.6382 & 0.0828 \\
\hline-1.275 & -3.596 & -1.799 & -0.2986 & -0.5276 & -0.4576 & 0.0857 \\
\hline 2.583 & 3.471 & 3.561 & -2.189 & -3.079 & -3.230 & 0.1229 \\
\hline 3.718 & 5.109 & 5.277 & -1.870 & -1.684 & -1.725 & 0.1232 \\
\hline 0.9849 & -1.243 & 1.121 & 0.4462 & 1.478 & 0.4831 & 0.1246 \\
\hline 5.734 & 4.377 & 5.733 & -0.9031 & -0.2398 & 0.2446 & 0.1262 \\
\hline 5.809 & 4.223 & 5.263 & -0.3537 & -0.3530 & -1.229 & 0.1328 \\
\hline 6.161 & 4.209 & 5.361 & -0.4353 & -0.2030 & -0.4448 & 0.1364 \\
\hline 5.852 & 5.521 & 4.580 & -0.0429 & -0.1258 & -0.7442 & 0.1398 \\
\hline 6.070 & 5.767 & 4.694 & 0.2238 & 0.2303 & 0.3625 & 0.1410 \\
\hline 5.568 & 4.914 & 4.844 & -0.1332 & -0.5524 & -1.413 & 0.1422 \\
\hline 5.177 & 4.151 & 5.738 & -0.8735 & -0.4692 & -1.234 & 0.1434 \\
\hline 1.719 & 0.0472 & -0.2643 & 0.2274 & -0.0260 & -0.0516 & 0.1446 \\
\hline 2.555 & 4.055 & 1.465 & 1.798 & -0.9142 & 1.256 & 0.1590 \\
\hline-1.332 & -1.942 & -2.359 & -0.6569 & -0.9574 & -1.164 & 0.1593 \\
\hline 0.7485 & 1.643 & -0.2211 & 0.0430 & 0.0195 & -0.5323 & 0.1894 \\
\hline 1.870 & 2.051 & 2.113 & 2.226 & 1.752 & 0.2477 & 0.1934 \\
\hline 1.590 & 4.243 & 1.968 & 0.8727 & -1.124 & 1.006 & 0.2044 \\
\hline-1.026 & 3.253 & 0.2169 & 0.1767 & 0.0083 & -0.2709 & 0.2254 \\
\hline 2.261 & 2.692 & 2.076 & 2.313 & 0.3841 & 0.8505 & 0.2281 \\
\hline 3.500 & 2.403 & 2.367 & 1.631 & 0.5951 & 0.6044 & 0.2340 \\
\hline 0.0375 & 0.1406 & 0.9122 & 0.0104 & 0.0386 & 0.4382 & 0.2580 \\
\hline-0.3547 & 3.084 & -0.5894 & 0.0129 & -0.0005 & 0.0230 & 0.2839 \\
\hline
\end{tabular}

Table 1 The remaining 46 elements of $\mathscr{S}^{c}$ beside the one given in Eq. 25 ordered with respect to the elastic strain energy density $U /(A L)$. 